\title{
NCAPG promotes the progression of lung adenocarcinoma via the TGF- $\beta$ signaling pathway
}

Yun $\mathrm{Wu}^{1 \dagger}$, Ying Lin ${ }^{1 \dagger}$, Junfan Pan ${ }^{1}$, Xunwei Tu ${ }^{1,2}$, Yiquan Xu ${ }^{1}$, Hongru Li $\mathrm{Li}^{1,2,3^{*}}$ and Yusheng Chen ${ }^{1,2,3^{*}}$

\begin{abstract}
Background: Lung cancer has the highest case fatality rate among cancers because of uncontrolled proliferation and early metastasis of cancer cells in the lung tissue. This study aimed to clarify the role of the non-SMC condensin I complex, subunit G (NCAPG) in lung adenocarcinoma (LUAD), explore the mechanisms of its progression, and lay the foundation for the search for new biological markers.
\end{abstract}

Methods: We analyzed overlapping differentially expressed genes (DEGs) from three datasets; a protein-protein interaction (PPI) network was subsequently constructed and analyzed using Cytoscape. We then selected NCAPG for validation because of its poor prognosis and because it has not been sufficiently studied in the context of LUAD. Immunohistochemical analysis was used to detect the expression of NCAPG in LUAD tissues, and the relationships between NCAPG and clinical parameters were analyzed. In vitro and in vivo experiments were conducted to verify the role of NCAPG in LUAD. Finally, we studied the specific mechanism of action of NCAPG in LUAD.

Results: Through comprehensive analysis of the GSE43458, GSE75037, and The Cancer Genome Atlas databases, we identified 517 overlapping DEGs. Among them, NCAPG was identified as a hub gene. Immunohistochemical analysis revealed that NCAPG was strongly associated with the clinical stage, M-classification, and N-classification. Univariate and multivariate Cox regression analyses indicated that NCAPG was a prognostic risk factor for LUAD, while the in vitro experiments showed that NCAPG overexpression promoted proliferation, migration, invasion, and epithelial-mesenchymal transition. Furthermore, knockdown of NCAPG inhibited LUAD progression, both in vitro and in vivo. Mechanistically, NCAPG overexpression increased $\mathrm{p}$-Smad2 and p-Smad3 expressions in the transforming growth factor $\beta$ (TGF- $\beta$ ) signaling pathway. Additionally, rescue experiments indicated that TGF- $\beta$ signaling pathway inhibitors could restore the effect of NCAPG overexpression in LUAD cells.

Conclusions: NCAPG may promote proliferation and migration via the TGF- $\beta$ signaling pathway in LUAD.

Keywords: Lung adenocarcinoma, NCAPG, TGF- $\beta$ signaling pathway, Prognostic biomarkers

*Correspondence: muzi131122@163.com; cysktz@fimu.edu.cn

${ }^{\dagger}$ Yun Wu and Ying Lin contributed equally to this work

${ }^{2}$ Department of Pulmonary and Critical Care Medicine, Fujian Provincial Hospital, Fuzhou 350001, China

Full list of author information is available at the end of the article

\begin{abstract}
Background
Lung cancer has the highest case fatality rate among all cancers because of uncontrolled proliferation and early metastasis of cancer cells in the lung tissue [1,2]. Lung cancer includes small and non-small cell lung carcinoma; non-small cell lung cancer includes adenocarcinoma, squamous cell carcinoma, and large cell carcinoma, accounting for approximately $85 \%$ of all lung cancer cases
\end{abstract} original author(s) and the source, provide a link to the Creative Commons licence, and indicate if changes were made. The images or other third party material in this article are included in the article's Creative Commons licence, unless indicated otherwise in a credit line to the material. If material is not included in the article's Creative Commons licence and your intended use is not permitted by statutory regulation or exceeds the permitted use, you will need to obtain permission directly from the copyright holder. To view a copy of this licence, visit http://creativecommons.org/licenses/by/4.0/. The Creative Commons Public Domain Dedication waiver (http://creativeco mmons.org/publicdomain/zero/1.0/) applies to the data made available in this article, unless otherwise stated in a credit line to the data. 
[3]. The survival of patients with lung adenocarcinoma (LUAD) has increased with the advent of combined surgery, radiotherapy, and chemotherapy; however, the survival rate remains poor, as many patients are diagnosed at an advanced stage $[4,5]$. Hence, it is essential to identify the underlying molecular mechanisms and significant prognostic biomarkers of LUAD.

Through the integrated analysis of the GSE43458, GSE75037, and The Cancer Genome Atlas (TCGA) databases for LUAD, we identified 517 overlapping differentially expressed genes (DEGs). Hub genes were selected by constructing a protein-protein interaction (PPI) network; we then analyzed the gene ontology (GO) and Kyoto Encyclopedia of Genes (KEGG) pathways of these genes. Among the hub genes, non-SMC condensin I complex subunit G (NCAPG)-a chromosome-condensed protein related to mitosis, with a relative molecular weight of approximately $114 \mathrm{kDa}$-was selected to be associated with a poor prognosis.

Previous researchers have found that NCAPG plays a vital role in condensin activation and the stabilization of chromosomes during mitosis [6], and increasing evidence shows that NCAPG overexpression is positively correlated with liver [7-10], gastric [11, 12], breast [13, 14], and renal cancer [15]; however, the functional role of NCAPG in LUAD remains unclear. To verify this, we conducted in vitro and in vivo experiments to determine whether NCAPG affects the malignant behavior of LUAD.

\section{Methods}

\section{Biological information analysis}

GSE43458 (including 80 LUAD and 30 adjacent normal lung tissues) and GSE75037 (including 83 LUAD and 83 adjacent normal lung tissues) data were obtained from the GEO database; the GEO2R method of analysis was performed on the two GEO datasets. In addition, RNA sequencing data from the TCGA LUAD cohort were downloaded, containing 478 LUAD and 57 adjacent normal lung tissues. The "limma" package in $\mathrm{R}$ (version 3.6.2; $\mathrm{R}$ Foundation for Statistical Computing, Vienna, Austria) was utilized to identify the DEGs between LUAD and normal lung tissue samples; genes-with "adjusted $P$-value $<0.05$ " and " $|\log \mathrm{FC}| \geq 1$ " as basic parameterswere selected as DEGs from each dataset.

The R package, clusterProfiler [16] - able to analyze and visualize the functional profiles of the genome coordinates-was used to perform GO functional enrichment and KEGG pathway enrichment analysis. PPI analysis was performed using STRING (https://string-db. org/) to identify DEGs; the results were visualized using Cytoscape [17], while hub genes were identified using CytoHubba [18] (degree $\geq 40$ ).

To explore the possible molecular mechanisms of NCAPG in lung cancer cell lines, we downloaded and compiled RNA-seq data from 80 LUAD cell lines from the Cancer Cell Line Encyclopedia website [19]. We performed gene set enrichment analysis (GSEA) [20] on the data of the LUAD cell lines to further explore the signaling pathways of NCAPG.

The Comparative Toxicogenomics Database (CTD, http://ctdbase.org/) [21] is a website that helps improving understanding of the chemical-gene-disease relationship. In CTD, the links between chemical-gene-disease are extracted from the literature. Furthermore, a network between chemotherapeutic drugs and NCAPG was constructed based on CTD and visualized using Cytoscape.

\section{Patients and tissue samples}

We collected 292 paraffin-embedded primary LUAD cancer tissues from patients at the Fujian Provincial Hospital, Fujian Medical University. Samples were collected between January 2010 and January 2021. The Ethics Committee of Fujian Provincial Hospital approved the use of all samples (K2021-04-092). Patients with a precise diagnosis and complete clinical information, who had not previously received radiation or chemotherapy, were included.

\section{Immunohistochemical analysis}

The samples were prepared with a thickness of $0.5 \mu \mathrm{m}$, dewaxed, rehydrated, and incubated with NCAPG antibodies (1:200; Abcam, Cambridge, UK) and Ki67 antibodies (1:200; Abcam) in a wet box at $4{ }^{\circ} \mathrm{C}$. After removing the antibodies, the samples were incubated with HRP-conjugated secondary antibodies; diaminobenzidine (1:50) was then added, and the cells were washed with phosphate-buffered saline (PBS) after $1 \mathrm{~min}$.

Five fields of view were randomly selected for each slice and scored using a double-blind method by more than two experienced pathologists; the scores were based on the percentage of stained cells and degree of staining. Regarding the number of pigmented cells, 0 indicated that $0-10 \%$ of cells were stained, and $1,2,3$, and 4 respectively indicated that $11-25 \%, 26-50 \%, 51-75 \%$, and $>75 \%$ of cells were stained. Regarding the degree of staining, 0 indicated no staining, and 1,2 , and 3 
respectively indicated light yellow, yellow, and brownish yellow staining. The two scores were multiplied to obtain a final quantification for each sample. Staining intensity was graded as negative for scores $0-3$, weakly positive for scores 4-6, moderately positive for scores 7-9, and strongly positive for scores 10-12. Negative and weakly positive scores were considered to indicate low expression; conversely, moderately and strongly positive scores were considered to indicate high expression.

\section{Cell culture and infection}

The human bronchial epithelial and LUAD cell linesBEAS-2B, and PC9, A549, H827, H1299, and H1975were purchased from the cell bank of the Chinese Academy of Sciences in Shanghai, China; they were then cultured in RMPI-1640 medium with $10 \%$ fetal bovine serum and incubated at $37{ }^{\circ} \mathrm{C}$ with $5 \% \mathrm{CO}_{2}$. Cell lines were authenticated to be free of mycoplasma contamination. PC9 and A549 cells were infected with lentiviruses harboring NCAPG overexpression. NCAPG-targeting short hairpin RNA (shRNA) was synthesized by Hanheng Biology Co., Ltd. (Shanghai, China). The target sequences of shRNA were as follows: sh-NCAPG 1\#: 5'-GCTGTC AGAAAGCTGGCTTAT-3' and sh-NCAPG 2\#: 5'-GCT GAAACATTGCAGAAATGT-3'. RepSox was purchased from Selleck (Shanghai, China).

\section{Western blot assay}

The tissues and cells were lysed with a protease inhibitor cocktail for $20 \mathrm{~min}$ and then centrifuged to remove the supernatant. Protein concentration was quantified using a BCA kit; $30 \mu \mathrm{g}$ of lysate from each sample was then separated by SDS-polyacrylamide gel electrophoresis, then blotted onto polyvinylidene fluoride membranes. Next, the primary antibody was added after sealing with $3 \%$ bovine serum albumin. Detailed information regarding the antibodies used in this study is listed in Additional file 1: Table S1. After washing the primary antibody with PBS, the corresponding HRP-linked secondary antibody was added; enhanced chemiluminescence was added for detection.

\section{Cell Counting Kit 8 (CCK8) assay}

CCK8 (Beyotime Biotechnology, Shanghai, China) was used to evaluate cell proliferation. After lentivirus infection, CCK 8 reagent was added to the culture medium; the absorbance at $450 \mathrm{~nm}$ was measured after $24,48,72$, and $96 \mathrm{~h}$.

\section{Clone formation experiment}

Cells were trypsinized and inoculated in a six-well plate at approximately 1000 cells/well. The plate was frequently observed, and the culture was stopped when clones were visible; the plate was then fixed with methanol, stained with crystal violet, and quantified.

\section{Wound healing assay}

Cells were cultured in a six-well plate at a cell density of $5 \times 10^{5}$ cells/well. When the cells reached $90 \%$ confluence, a straight line was vertically drawn at the bottom of the well using the tip of a $10 \mu \mathrm{L}$ pipette. Debris was removed and cultured in serum-free RPMI-1640 medium, and photographs were acquired at 0 and $48 \mathrm{~h}$.

\section{Transwell migration assay}

In the cell migration experiment, $200 \mu \mathrm{L}$ of cell suspension $\left(1 \times 10^{5} / \mathrm{mL}\right)$ with serum-free RPMI-1640 medium was added to the upper well, while $600 \mu \mathrm{L}$ medium containing $20 \%$ fetal bovine serum without cells was added to the lower chamber. After $24 \mathrm{~h}$, the upper chamber was carefully removed, washed, fixed with paraformaldehyde, and stained with crystal violet. The non-migrating cells in the upper chamber were then gently wiped off with a cotton swab; photographs were subsequently obtained. Five fields of view were randomly selected for counting.

In the invasion experiment, the upper chamber was precoated with Matrigel (BD Biosciences, San Jose, CA) before the cells were added; subsequent steps were consistent with those of the migration experiments.

\section{Xenograft tumor model}

Four- to five-week-old male BABL/c nude mice (14-18 g) were purchased from Slack Laboratory Animal Co., Ltd. (Shanghai, China). After lentivirus infection, PC9 cells $\left(5 \times 10^{6}\right)$-including sh-Control, sh-NCAPG $1 \#$, and shNCAPG 2\#-were injected into the right flanks of mice. Tumors were measured every 7 days, and their volumes were calculated as follows: (length $\times$ width $\left.^{2}\right) / 2$. On day 28 , the tumors were harvested for immunohistochemical analyses.

\section{Statistical analysis}

Statistical analyses were performed using SPSS 22.0 (IBM Corp., Armonk, NY). The association between gene expression and clinicopathological characteristics was analyzed using the chi-squared test, while the KaplanMeier method was used for the survival analysis. In 


\begin{tabular}{|l|l|l|l|l|}
\hline & & GSE75037 \\
\hline
\end{tabular}

addition, differences between two groups were analyzed using Student's t-test, while differences among three or more groups were analyzed using one-way analysis of variance; statistical significance was set at $\mathrm{p}<0.05$. The assays were performed independently at least three times.

\section{Results}

\section{Identification of DEGs in LUAD}

DEGs were determined by comparing LUAD and adjacent lung tissue samples. We identified 517 DEGs (Fig. 1, Additional file 2: Table S2) with an adjusted p-value $<0.05$, and $|\log F C| \geq 1$. GO functional, and KEGG pathway enrichment analyses were performed to analyze the functions and mechanisms of common DEGs. The enriched GO terms included biological-process (BP), cell-component (CC), and molecular-function (MF) ontologies (Fig. 2a). BP analysis was enriched in the extracellular matrix organization, extracellular structure organization, cell-substrate adhesion, and others. $\mathrm{CC}$ analysis was mainly enriched in collagen-containing extracellular matrix, cell-cell junctions, membrane raft, and others. MF analysis was mainly enriched in extracellular matrix structural constituents, glycosaminoglycan binding, cytokine binding, among others. Additionally, the KEGG pathway (Fig. 2b) was mainly enriched in several pathways, including the relaxin signaling pathway, cell adhesion molecules, complement, and coagulation cascades.

\section{NCAPG identified as a targeted gene associated with poor prognosis}

STRING was used to construct a PPI network from overlapping genes to identify the most important genes (Additional file 3: Fig. S1). The top 14 genes were screened using Cytoscape according to the CytoHubba ranking method (Fig. 3a, Table 1). GEPIA (http://gepia. cancer-pku.cn/) [22] was employed to study the prognosis of these hub genes, and it was found that high expression was associated with poorer overall survival (Additional file 4: Fig. S2). NCAPG represents one of these genes (Fig. 3b), which has not been well studied in LUAD; therefore, this gene was selected for further
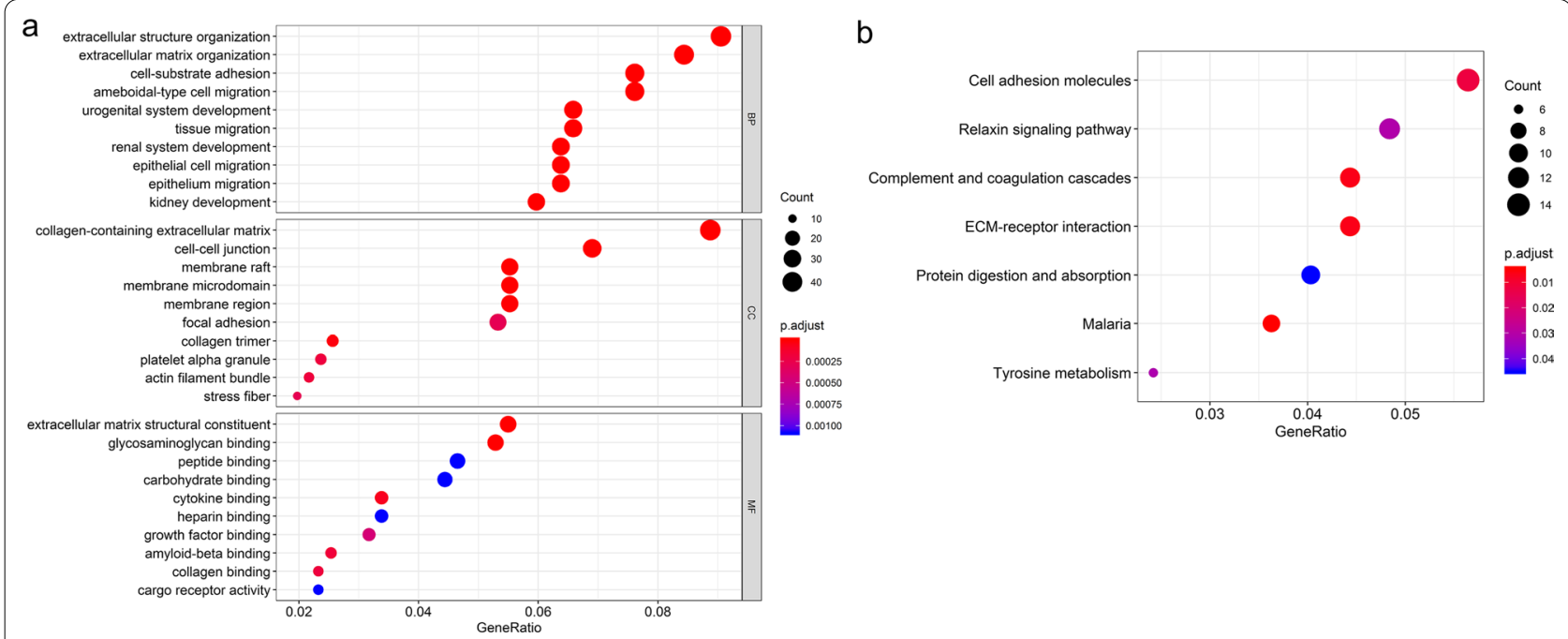

Fig. 2 GO and KEGG enrichment of 517 overlapping differentially-expressed genes. a The enriched GO terms consisted of biological-process, cell-component, and molecular-function ontologies. b The KEGG pathway was mainly enriched with seven pathways. ECM extracellular matrix, GO gene ontology, KEGG Kyoto Encyclopedia of Genes 



Fig. 3 NCAPG was identified as a targeted gene associated with poor prognosis. a The top 14 genes were screened using Cytoscape according to the CytoHubba ranking methods. b High expression of NCAPG was associated with poor overall survival. c NCAPG was upregulated in most cancers. d NCAPG was upregulated in tissue with lung adenocarcinoma in the TCGA cohort. e Increased NCAPG expression was associated with an advanced clinical TNM stage in the TCGA database. f The receiver operating characteristic area under the curve was 0.962 (95\% Cl 0.944-0.981; $\mathrm{p}$ <0.001). FPR false positive rate, TPM transcripts per million, TPR true positive rate

Table 1 Top 14 hub genes with higher degree

\begin{tabular}{llll}
\hline Rank & Gene symbol & Gene description & Degree \\
\hline 1 & CDK1 & Cyclin Dependent Kinase 1 & 45 \\
2 & KIF11 & Kinesin Family Member 11 & 44 \\
3 & CDC20 & Cell Division Cycle 20 & 43 \\
3 & CCNA2 & Cyclin A2 & 43 \\
3 & BUB1 & BUB1 Mitotic Checkpoint Serine/Threonine Kinase & 43 \\
6 & MELK & Maternal Embryonic Leucine Zipper Kinase & 42 \\
6 & TOP2A & DNA Topoisomerase II Alpha & 42 \\
6 & ASPM & Assembly Factor For Spindle Microtubules & 42 \\
9 & NCAPG & Non-SMC Condensin I Complex Subunit G & 41 \\
9 & CENPE & Centromere Protein E & 41 \\
11 & RRM2 & Ribonucleotide Reductase Regulatory Subunit M2 & 40 \\
11 & DLGAP5 & DLG Associated Protein 5 & 40 \\
11 & TTK & TTK Protein Kinase & 40 \\
11 & TPX2 & TPX2 Microtubule Nucleation Factor & 40 \\
\hline
\end{tabular}



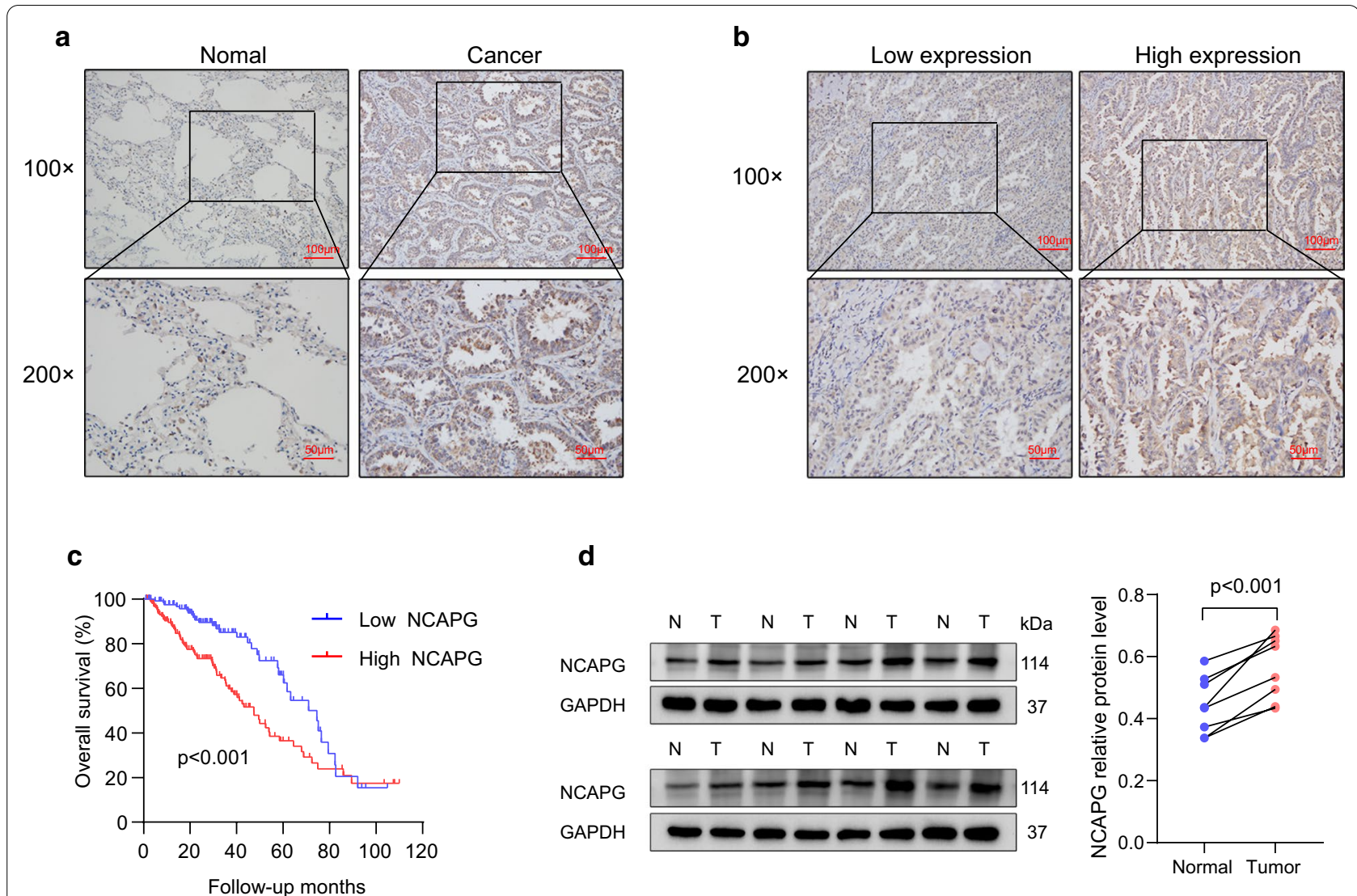

Fig. 4 High NCAPG expression was associated with poor prognosis in our cohort. a Immunohistochemical staining revealed that NCAPG appears brown in lung adenocarcinoma (LUAD) tissue. b Representative photographs of low and high expression of NCAPG in LUAD tissue. c Kaplan-Meier analysis indicated that patients with LUAD and higher NCAPG expression had shorter survival. $\mathbf{d}$ Western blot analysis showed that NCAPG was overexpressed in human primary LUAD tissues compared with adjacent lung tissues

analysis. Furthermore, NCAPG expression was upregulated in most cancers (Fig. 3c).

By analyzing the expression of NCAPG in patients with LUAD through the TCGA database, we found that NCAPG expression was upregulated $(\mathrm{p}<0.001$, Fig. $3 \mathrm{~d}$ ) in LUAD tissue samples compared with adjacent normal tissue samples; additionally, increased NCAPG expression was associated with advanced clinical TNM stages (Fig. 3e). The receiver operating characteristic area under the curve was 0.962 (95\% CI 0.944-0.981; p $<0.001$; Fig. 3f), indicating that NCAPG has high sensitivity and specificity for the diagnosis of LUAD.

\section{High NCAPG expression was associated with poor prognosis}

To verify the expression of NCAPG in LUAD, immunohistochemical analyses were used to detect the expression of NCAPG in 292 cases of paraffin-embedded LUAD tissues. Immunohistochemical staining showed that NCAPG appeared brown in the LUAD tissues (Fig. 4a).

Table 2 lists the demographics, clinicopathological characteristics, and tumor characteristics of the 292 patients with LUAD, including 142 female (48.6\%) and 150 male $(51.4 \%)$ patients aged $33-86$ years. Postoperative pathological staging identified 150 cases $(51.4 \%)$ at stage I, 72 cases $(24.7 \%)$ at stage II, 51 cases $(17.5 \%)$ at stage III, and 19 cases (6.5\%) at clinical stage IV of the 
Table 2 Clinicopathological characteristics of patient samples and expression of NCAPG in LUAD

\begin{tabular}{lc}
\hline Clinical characteristics & Number of cases (\%) \\
\hline Age (y) & \\
$\geq 65$ & $160(54.8)$ \\
$<65$ & $132(45.2)$ \\
Sex & \\
Male & $150(51.4)$ \\
Female & $142(48.6)$ \\
Clinical stage & \\
I & $150(51.4)$ \\
II & $72(24.7)$ \\
III & $51(17.5)$ \\
IV & $19(6.5)$ \\
T classification & \\
T1 & $88(30.1)$ \\
T2 & $164(56.2)$ \\
T3 & $24(8.2)$ \\
T4 & $16(5.5)$ \\
N classification & \\
N0 & $184(63.0)$ \\
N1 & $63(21.6)$ \\
N2 & $44(15.1)$ \\
N3 & $1(0.3)$ \\
Metastasis & \\
No & $273(93.5)$ \\
Yes & $19(6.5)$ \\
Low expression & \\
High expression & $128(43.8)$ \\
\hline Tfor extent of & $164(56.2)$ \\
\hline
\end{tabular}

T for extent of the primary tumor; $\mathrm{N}$ for involvement of lymph nodes

T1: Tumor $\leq 3 \mathrm{~cm}$; T2: Tumor $>3$ but $\leq 5 \mathrm{~cm}$ or tumor involving visceral pleura, main bronchus (not carina), atelectasis to hilum; T3: Tumor $>5$ but $\leq 7 \mathrm{~cm}$ or invading chest wall, pericardium, phrenic nerve or or separate tumor nodule(s) in the same lobe; T4: Tumor $>7 \mathrm{~cm}$ or tumor invading: mediastinum, diaphragm, heart, great vessels, recurrent laryngeal nerve, carina, trachea, esophagus, spine or tumor nodule(s) in a different ipsilateral lobe

N0: No regional node metastasis; $\mathrm{N} 1$ : Metastasis in ipsilateral pulmonary or hilar nodes; N2: Metastasis in ipsilateral mediastinal/subcarinal nodes; N3: Metastasis in contralateral mediastinal/hilar, or suprclavicular nodes

disease. The NCAPG protein level was overexpressed in $164(56.2 \%)$ samples, and low in $128(43.8 \%)$ samples (Fig. 4b).

The relationship between NCAPG and the clinicopathological parameters of LUAD was also analyzed. NCAPG expression was strongly correlated with N-classification $(\mathrm{p}=0.006), \quad$ M-classification $(\mathrm{p}=0.011)$, and clinical stage $(\mathrm{p}=0.004)$, as shown in Table 3; no correlations were observed regarding age $(\mathrm{p}=0.407)$, sex $(\mathrm{p}=0.556)$, or T-classification $(\mathrm{p}=0.091)$.
Table 3 Association between NCAPG expression and clinicopathologic characteristics of LUAD patients

\begin{tabular}{|c|c|c|c|}
\hline \multirow[t]{2}{*}{ Clinical characteristics } & \multicolumn{2}{|l|}{ NCAPG } & \multirow[t]{2}{*}{$p$-value } \\
\hline & Low expression & $\begin{array}{l}\text { High } \\
\text { expression }\end{array}$ & \\
\hline \multicolumn{4}{|l|}{ Age (y) } \\
\hline$\geq 65$ & 74 & 86 & 0.407 \\
\hline$<65$ & 54 & 78 & \\
\hline \multicolumn{4}{|l|}{ Sex } \\
\hline Male & 63 & 87 & 0.556 \\
\hline Female & 65 & 77 & \\
\hline \multicolumn{4}{|l|}{ Clinical stage } \\
\hline । & 79 & 71 & 0.004 \\
\hline$\|$ & 28 & 44 & \\
\hline III \& IV & 21 & 49 & \\
\hline \multicolumn{4}{|l|}{ T classification } \\
\hline $\mathrm{T} 1$ & 47 & 41 & 0.091 \\
\hline T2 & 66 & 98 & \\
\hline T3 \& T4 & 15 & 25 & \\
\hline \multicolumn{4}{|l|}{ N classification } \\
\hline NO & 92 & 92 & 0.006 \\
\hline N1 \& N2 \& N3 & 36 & 72 & \\
\hline \multicolumn{4}{|l|}{ Metastasis } \\
\hline No & 125 & 148 & 0.011 \\
\hline Yes & 3 & 16 & \\
\hline
\end{tabular}

T for extent of the primary tumor; $\mathrm{N}$ for involvement of lymph nodes

$\mathrm{T} 1$ : Tumor $\leq 3 \mathrm{~cm} ; \mathrm{T} 2$ : Tumor $>3$ but $\leq 5 \mathrm{~cm}$ or tumor involving visceral pleura, main bronchus (not carina), atelectasis to hilum; T3: Tumor $>5$ but $\leq 7 \mathrm{~cm}$ or invading chest wall, pericardium, phrenic nerve or or separate tumor nodule(s) in the same lobe; T4:Tumor $>7 \mathrm{~cm}$ or tumor invading: mediastinum, diaphragm, heart, great vessels, recurrent laryngeal nerve, carina, trachea, esophagus, spine or tumor nodule(s) in a different ipsilateral lobe

N0: No regional node metastasis; N1: Metastasis in ipsilateral pulmonary or hilar nodes; N2: Metastasis in ipsilateral mediastinal/subcarinal nodes; N3: Metastasis in contralateral mediastinal/hilar, or suprclavicular nodes

Kaplan-Meier analysis indicated that among patients with LUAD, those with higher NCAPG expression had shorter survival than those with lower NCAPG expression $(\mathrm{p}<0.001$; Fig. $4 \mathrm{c})$, while univariate and multivariate Cox regression analyses showed that NCAPG was an independent prognostic factor in patients with LUAD (Table 4). Furthermore, we analyzed eight paired tumor and non-cancerous lung tissues to detect the expression of NCAPG. Western blot analysis showed that NCAPG was over expressed in human primary LUAD tissues compared with adjacent lung tissues (Fig. 4d). 
Table 4 Univariate and multivariate Cox regression analyses of various prognostic parameters in patients with LUAD

\begin{tabular}{|c|c|c|c|c|c|c|}
\hline \multirow[t]{2}{*}{ Parameter } & \multicolumn{3}{|c|}{ Univariate analysis } & \multicolumn{3}{|c|}{ Multivariate analysis } \\
\hline & HR & $95 \% \mathrm{Cl}$ & $\mathbf{P}$ & HR & $95 \% \mathrm{Cl}$ & $\mathbf{P}$ \\
\hline Age (y) & 1.030 & $0.697-1.523$ & 0.882 & & & \\
\hline Sex & 0.963 & $0.654-1.418$ & 0.848 & & & \\
\hline Clinical stage & 1.834 & $1.528-2.201$ & $<0.001$ & 2.200 & $1.092-2.591$ & 0.002 \\
\hline T classification & 1.645 & $1.304-2.076$ & $<0.001$ & 1.121 & $0.861-1.459$ & 0.396 \\
\hline N classification & 1.931 & $1.559-2.393$ & $<0.001$ & 0.977 & $0.656-1.454$ & 0.908 \\
\hline Metastasis & 2.136 & $1.135-4.020$ & 0.019 & 0.358 & $0.114-1.130$ & 0.080 \\
\hline NCAPG & 2.048 & $1.348-3.111$ & $<0.001$ & 1.682 & $1.092-2.591$ & 0.018 \\
\hline
\end{tabular}

$\mathrm{T}$ for extent of the primary tumor; $\mathrm{N}$ for involvement of lymph nodes

\section{NCAPG promoted proliferation and metastasis in LUAD cells}

Through western blot analyses, we verified that NCAPG expression in LUAD cell lines was upregulated compared with that in BEAS-2B cell lines (Fig. 5a). To further validate the role of NCAPG in LUAD cells, we ectopically overexpressed NCAPG in PC9 and A549 LUAD cells (Fig. 5b). CCK8 assays, colony formation, wound healing, and transwell assays were performed to identify the role of NCAPG in the progression, invasion, and migration of LUAD (Fig. 5c-f); interestingly, it was found to induce cell progression, migration, and invasion in PC9 and A549 cell lines. Additionally, we constructed cells with stable NCAPC knockdown using shRNAs (Fig. 6a); silencing of NCAPG led to the inhibition of cell proliferation, invasion, and migration (Fig. 6b-e). Epithelial-mesenchymal transition (EMT) marker genes were further detected with western blot analysis. We found that when NCAPG was overexpressed, the expression of N-cadherin, vimentin, and Snail increased, while the expression of E-cadherin decreased (Fig. 5g); conversely, when NCAPG was knocked down, the expression of N-cadherin, vimentin, and Snail decreased, while the expression of E-cadherin increased (Fig. 6f). These results indicate that NCAPG plays a key role in the progression of LUAD.

\section{NCAPG modulated the tumorigenesis of LUAD}

To verify the results of the in vivo experiments, we used PC9 cells in male BALB/C nude mice to establish a xenograft model. As shown in Fig. 7a, compared with the cells infected with the vector, the ability of NCAPG-silenced cells to form tumors in nude mice was significantly reduced, indicated by the weight and growth curve of the tumor (Fig. 7b, c). NCAPG was detected in tumors using immunohistochemical staining, revealing that tumors formed by sh-NCAPG cells were significantly lower than those of sh-Ctrl cells (Fig. 7d). In tumors formed by shNCAPG cells, the expression of Ki-67 was decreased compared with the sh-Ctrl group (Fig. 7e). Ultimately, NCAPG was found to play an oncogenic role in LUAD.

\section{NCAPG promoted LUAD progression and proliferation through the transforming growth factor $\beta$ (TGF- $\beta$ ) signaling pathway}

GSEA enrichment analysis was performed to explore the pathogenic mechanism of NCAPG in LUAD, revealing that the abnormal expression of NCAPG was related to the TGF- $\beta$ signaling pathway (Fig. 8a). We detected the main proteins involved in the TGF- $\beta$ signaling pathway, and the results are shown in Fig. 8b. After overexpressing NCAPG, the phosphorylation levels of Smad2 and Smad3 increased, whereas the total Smad2 and Smad3 levels remained constant. After NCAPG knockdown, the phosphorylation levels of Smad2 and Smad3 significantly decreased; however, no change in the total Smad2 and Smad3 levels was observed.

\section{Inhibiting TGF- $\beta$ reduced progression of LUAD induced by overexpressing NCAPG}

To confirm that NCAPG functions via the TGF- $\beta$ signaling pathway, the TGF- $\beta$ inhibitor RepSox was added to the culture medium after NCAPG overexpression. Colony formation and transwell assays were used to determine proliferation, migration, and invasion ability. Interestingly, cell proliferation, migration, and invasion ability were eliminated in the NCAPG + RepSox group 


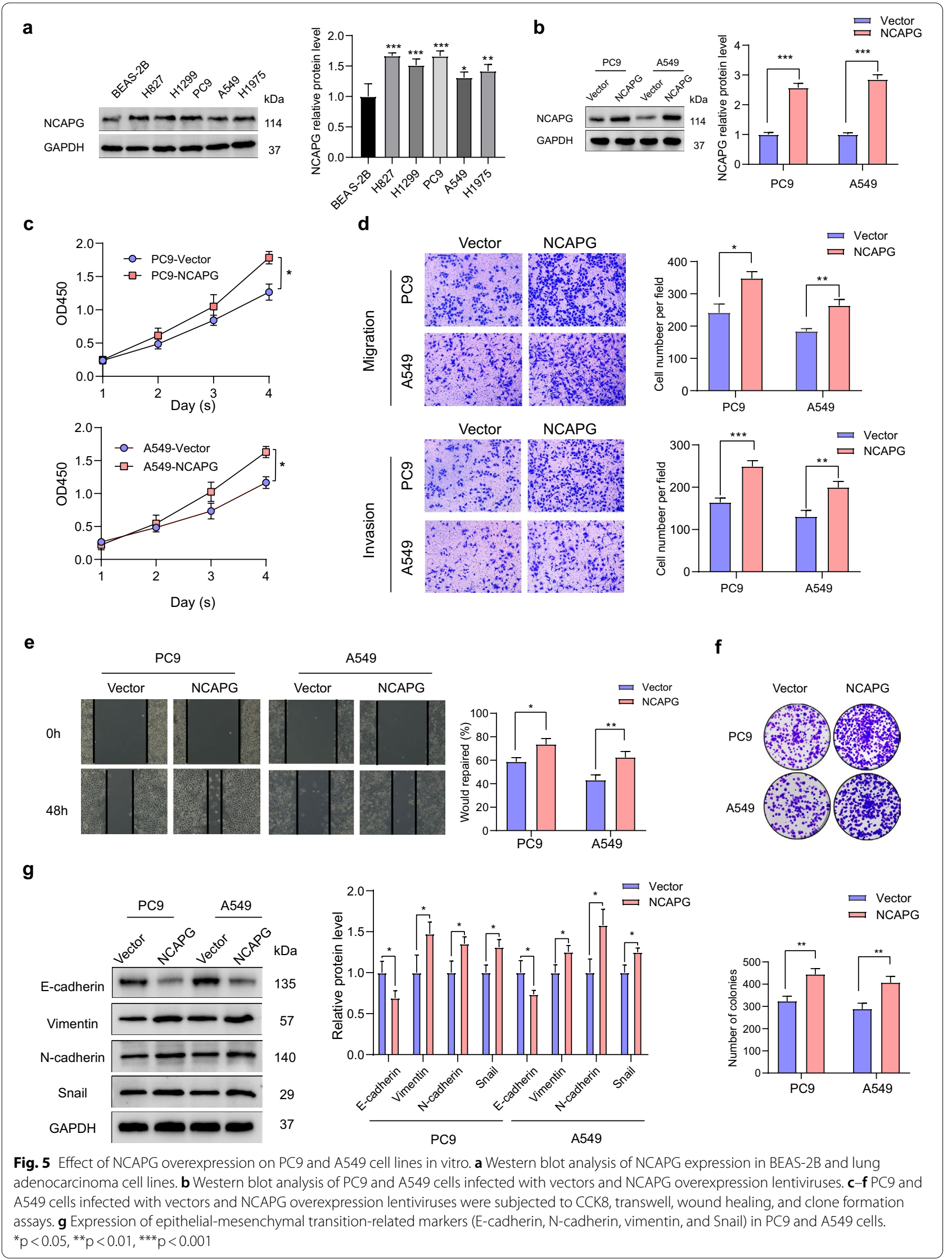


(Fig. 8c, d); additionally, western blot analysis showed that NCAPG + RepSox inhibited EMT induced via NCAPG overexpression, characterized by upregulation of E-cadherin, and downregulation of N-cadherin, vimentin, and Snail (Fig. 8e). Simultaneously, NCAPG + RepSox weakened the activation of the TGF- $\beta /$ Smad signaling pathway through overexpression of NCAPG (Fig. 8f). These results suggest that inhibition of TGF- $\beta$ reduces the tumorigenic effect of NCAPG in LUAD.

\section{Identify chemotherapeutic drugs targeting NCAPG}

To gain further insight into the gene-targeted therapy, a network between chemotherapeutic drugs and NCAPG was constructed based on CTD. As shown in Fig. 9, the network showed that fluorouracil, azathioprine, palbociclib, doxorubicin, dasatinib, and zoledronic acid could reduce the expression of NCAPG.

\section{Discussion}

Data mining can help us obtain more information regarding the mechanisms of tumor occurrence and development. Many oncogenes and tumor suppressor genes can be evidenced by multi-omics analyses from tumor tissues, and these genes are closely related to the prognosis of patients. By examining the copy number changes and mutation of protein-coding genes in 100 breast cancer tissues, Stephens et al. [23] found at least 40 oncogenes related to poor prognosis. Liu et al. [24] synthesized data from 2778 patients from 15 public data sets to identify reliable biomarkers for the prognosis and distant metastasis risk of colorectal cancer. Bioinformatics analyses were applied in our study by integrating three databases, i.e., GSE43458, GSE75037, and TCGA, and 517 DEGs were identified. Among the common DEGs, 14 hub genes were screened using CytoHubba. NCAPG was found upregulated and associated with poor survival in patients with LUAD.

The expression of NCAPG has been reported to be associated with tumor progression. Gong et al. [7] performed immunohistochemical analyses in 90 hepatocellular carcinoma (HCC) and adjacent tissues; the results indicated that the expression of NCAPG was higher in $85 \mathrm{HCC}$ tissues than in non-HCC tissues. The group's in vitro experiments demonstrated that NCAPG promoted the proliferation and metastasis of $\mathrm{HCC}$ cells by activating the PI3K/AKT/FOXO4 pathway. Another study reported that NCAPG was significantly overexpressed in trastuzumab-resistant HER2+ breast cancer samples and that the upregulation of NCAPG was associated with poor survival and recurrence in patients with HER2+ breast cancer [13]; thus, to further determine whether NCAPG promotes LUAD progression, we designed a series of studies. Our findings provide evidence that NCAPG is both significantly upregulated in LUAD and associated with poor survival. Additionally, NCAPG overexpression promoted LUAD cell proliferation, invasion, and migration; therefore, we speculate that NCAPG may be an oncogene in LUAD and could serve as a potential biological target for the diagnosis of LUAD.

EMT can induce the escape and apoptosis of tumor cells, as well as promote migration and infiltration; once tumor cells undergo EMT, the possibility of metastasis increases [25]. The most significant changes in EMT are the decrease or absence of E-cadherin and increase of $\mathrm{N}$-cadherin, vimentin, and Snail. After EMT is activated, the tumor epithelial cells lose cell polarity and intercellular adhesion and gain migratory and invasive characteristics; they then change to mesenchymal cells, thereby promoting tumor cell metastasis. Currently, there is a general consensus that EMT is critical for modulating drug resistance, cancer invasion, and metastasis [26, 27]. Our study found that the overexpression of NCAPG led to upregulation of $\mathrm{N}$-cadherin, vimentin, and Snail, while E-cadherin was inhibited, proving that NCAPG promoted EMT in LUAD cells. Conversely, the downregulation of NCAPG led to a decrease in N-cadherin, vimentin, and Snail, and an increase in E-cadherin. We believe that NCAPG aggravates migration and invasion by improving EMT in LUAD.

We found that NCAPG was positively correlated with the TGF- $\beta$ signaling pathway-as shown by GSEAand it is well accepted that EMT can be induced by the TGF- $\beta$ signaling pathway $[28,29]$. TGF- $\beta$ is an inhibitory factor in precancerous cells, while in cancer cells, it plays a role in promoting metastasis. Tumor cells can secrete large amounts of TGF- $\beta$, promote tumor invasion and

(See figure on next page.)

Fig. 6 Effect of NCAPG knocking down on PC9 and A549 cell lines in vitro. a Western blot analysis of PC9 and A549 cells infected with sh-Ctrl, sh-NCAPG1\#, and sh-NCAPG2\# lentiviruses. b-e PC9 and A549 cells infected with sh-Ctrl, sh-NCAPG1\#, and sh-NCAPG2\# lentiviruses were subjected to CCK8, transwell, wound healing, and clone formation assays. $\mathbf{f}$ Expression of epithelial-mesenchymal transition-related markers (E-cadherin, $\mathrm{N}$-cadherin, vimentin, and Snail) in PC9 and A549 cells. ${ }^{*} p<0.05,{ }^{* *} p<0.01,{ }^{* *} p<0.001$. Ctrl control 



f
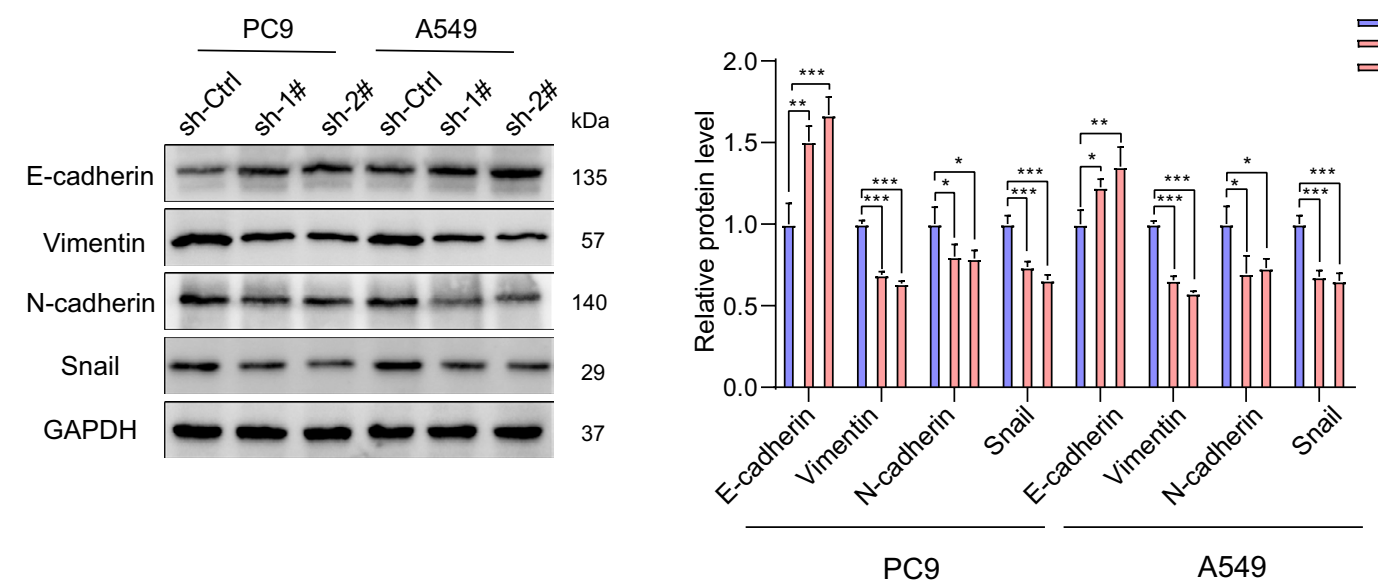

Fig. 6 (See legend on previous page.) 


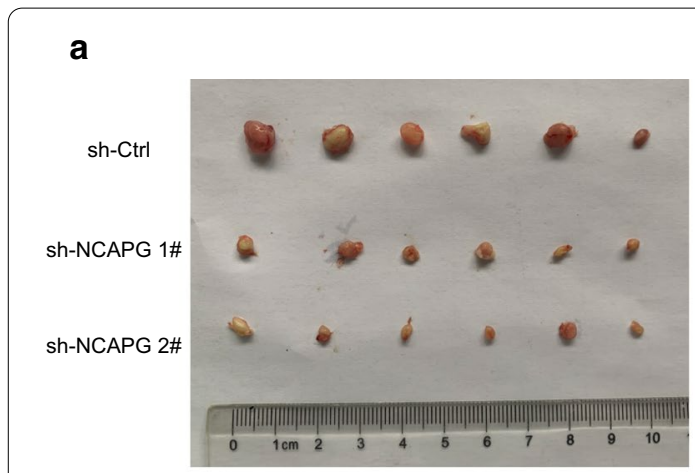

b
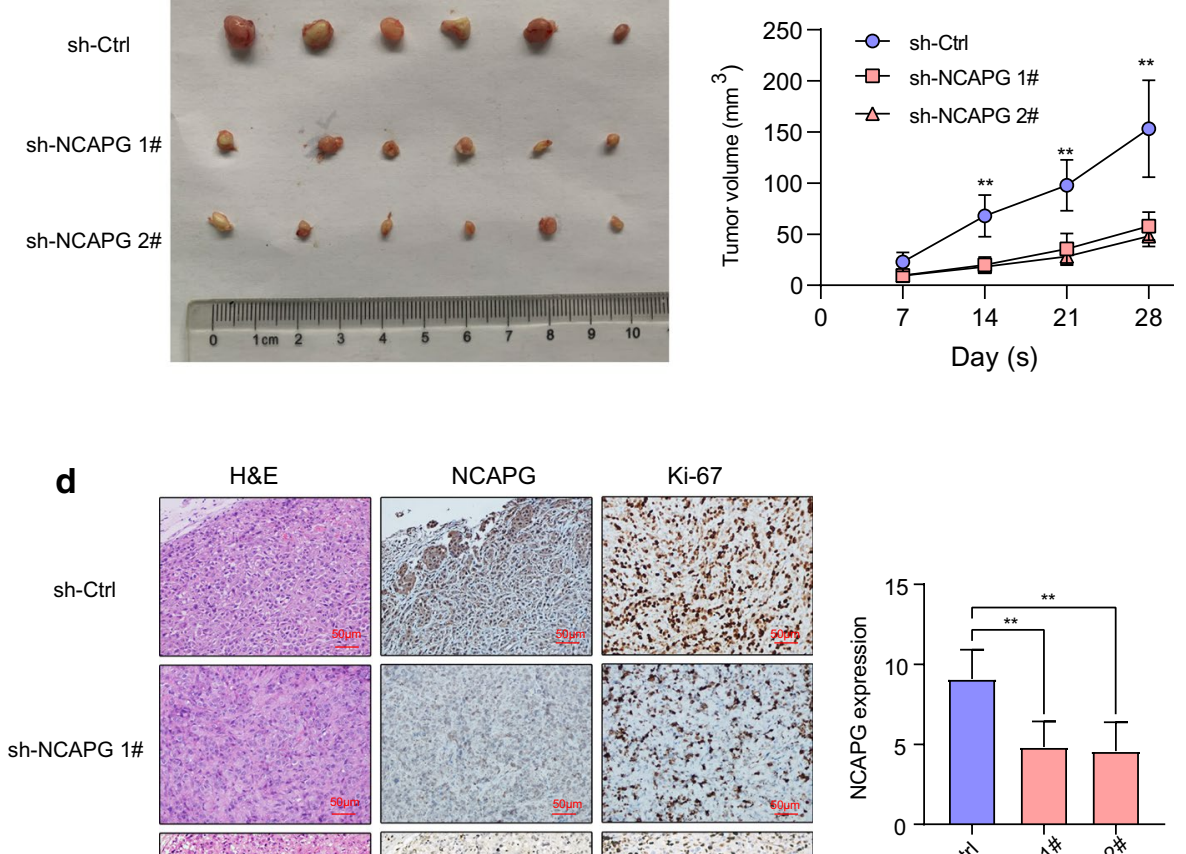

sh-NCAPG 2\#
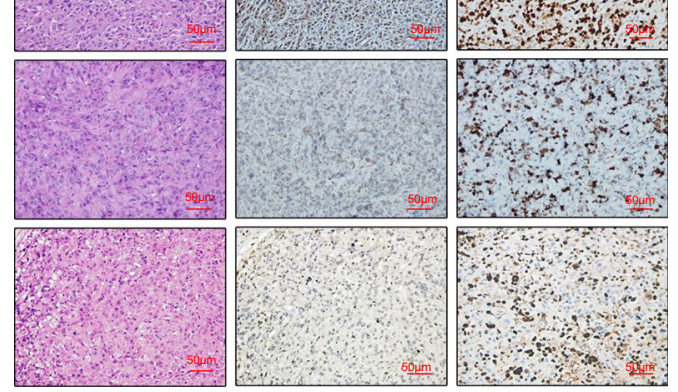

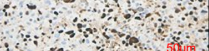

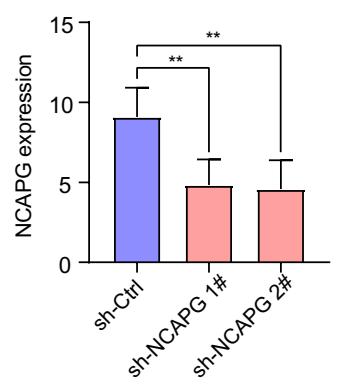

Fig. 7 Effect of NCAPC knockdown on the PC9 cell line in vivo. a Photographs of xenograft tumors. b Average weight of xenograft tumors. $\mathbf{c}$ Growth curve of xenograft tumors. $\mathbf{d}$ Hematoxylin and eosin staining and immunohistochemical staining of NCAPG and Ki-67 in xenograft tumors. e Quantitation of Ki-67 staining. ${ }^{*} p<0.05,{ }^{* *} p<0.01,{ }^{* * *} p<0.001$. Ctrl control

metastasis through the autocrine and paracrine pathways, and induce EMT, causing epithelial tumor cells to acquire an aggressive mesenchymal phenotype [30]. At the same time, secretion of intercellular adhesion molecules and metalloproteinases changes cause tumor metastasis [31].

Smad2 and Smad3 were found to mediate the classical TGF- $\beta /$ Smad signaling pathway. In the TGF- $\beta$ signaling pathway, the TGF- $\beta$ superfamily ligands bind to membrane T $\beta R I I$, subsequently phosphorylating $T \beta R I$ and forming complexes. The activated receptor complex further activates Smad2 and Smad3, and phosphorylated Smad2/Smad3 binds to Smad4; the Smad4 complex then enters the nucleus and regulates the expression of target genes with other transcription factors [32, 33]. In this study, we found that NCAPG promoted the progression of LUAD by positively regulating the TGF- $\beta$ pathway; additionally, a TGF- $\beta$ pathway inhibitor could reverse the effect of NCAPG overexpression on the progression of LUAD. It can therefore be concluded that NCAPG partially promotes LUAD progression through the TGF- $\beta$ pathway (Fig. 10).

In order to explore potential gene-targeted treatments against LUAD with NCAPG overexpression, we used CTD to construct a gene-drug network. Based on this network, we found that fluorouracil, azathioprine, palbociclib, doxorubicin, dasatinib, and zoledronic acid can reduce the expression of NCAPG, indicating that these drugs might be a therapeutic alternative for these patients. For instance, several studies have reported dasatinib as a growth inhibitor of lung cancer in vitro and in vivo [34, 35]. Pabocinil, mainly used for breast cancer treatment, was reported to be a potential therapeutic agent in patients with non-small cell lung cancer 


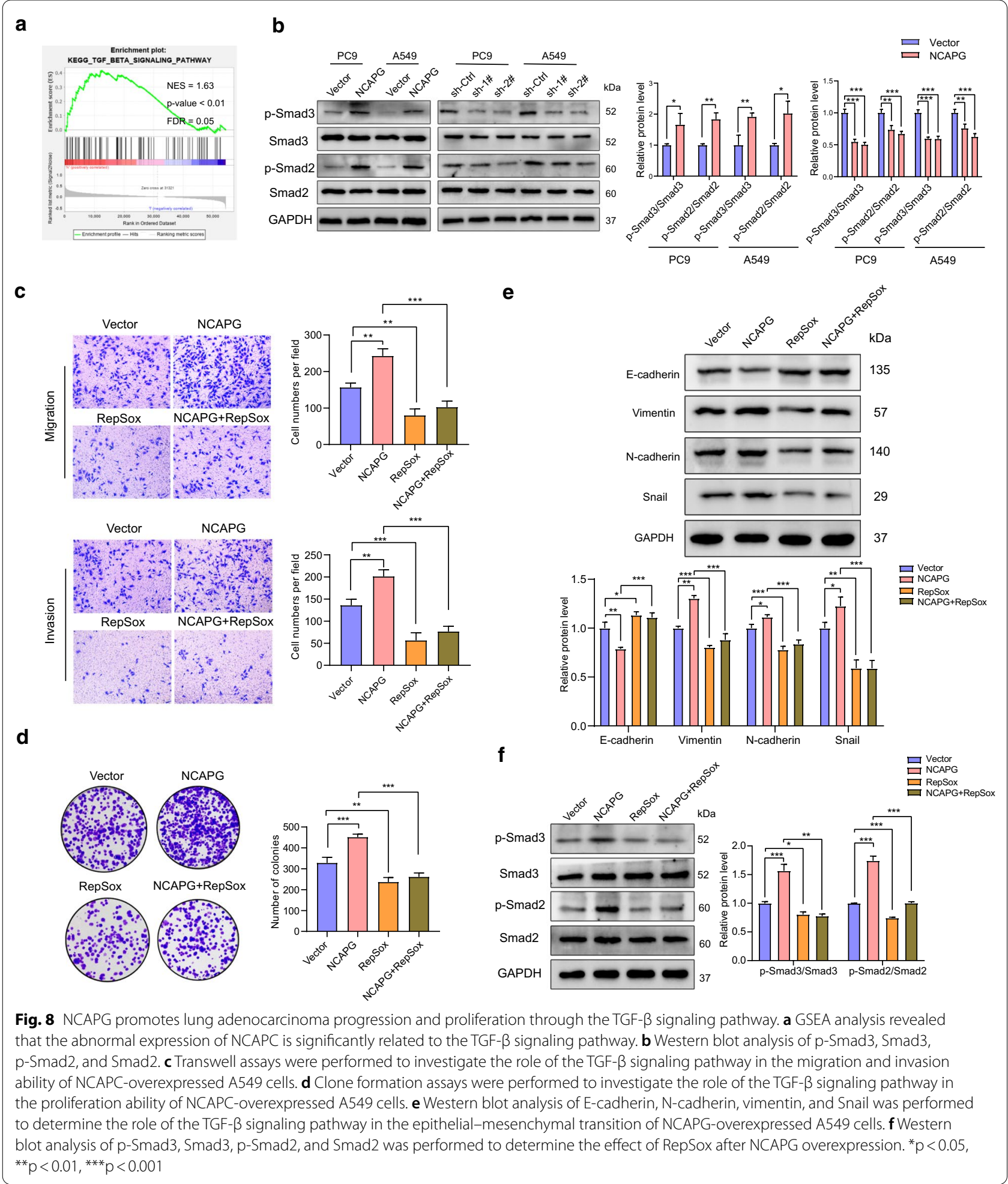

[36]. However, whether LUAD patients with overexpressing NCAPG can benefit from the use of these drugs still needs further experimental evidence.
There are some limitations to our study; first, further 


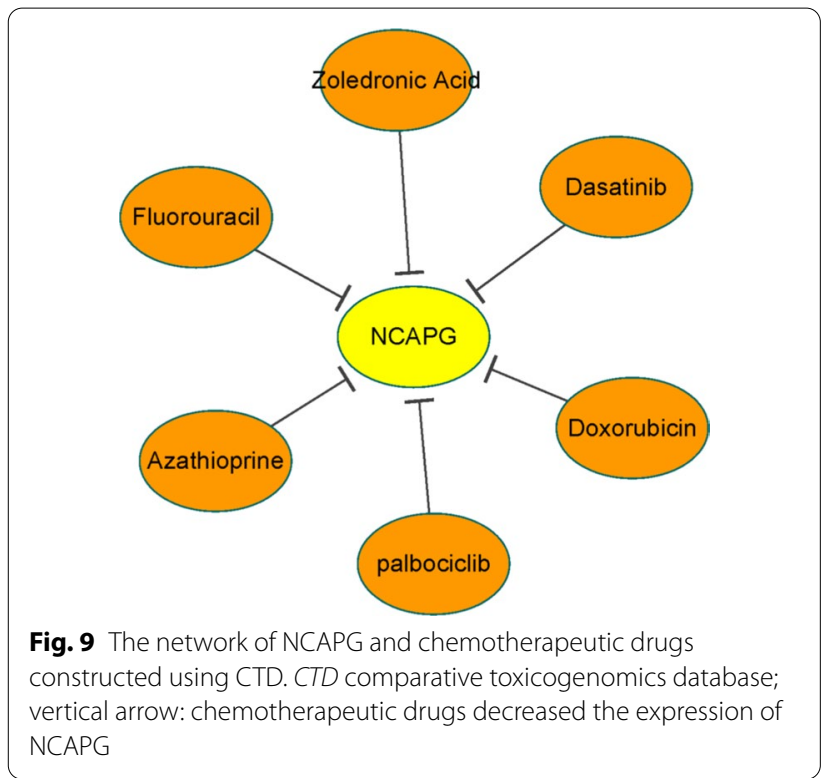

correlation with the TGF- $\beta$ signaling pathway-is limited. To a certain extent, this also limits our exploration of its molecular mechanism; therefore, our findings provide a foundation for further research on the downstream mechanisms of NCAPG-induced LUAD progression.

\section{Conclusion}

In summary, our study was the first to prove that NCAPG is an oncogene in LUAD. Moreover, NCAPGinduced LUAD may be related to the TGF- $\beta$ pathway. These findings provide new insights into the role of NCAPG regarding the occurrence and development of LUAD, which may further our understanding of the biological behavior of NCAPG, as well as help establish a potential treatment for LUAD.

vertical arrow: chemotherapeutic drugs decreased the expression of NCAPG

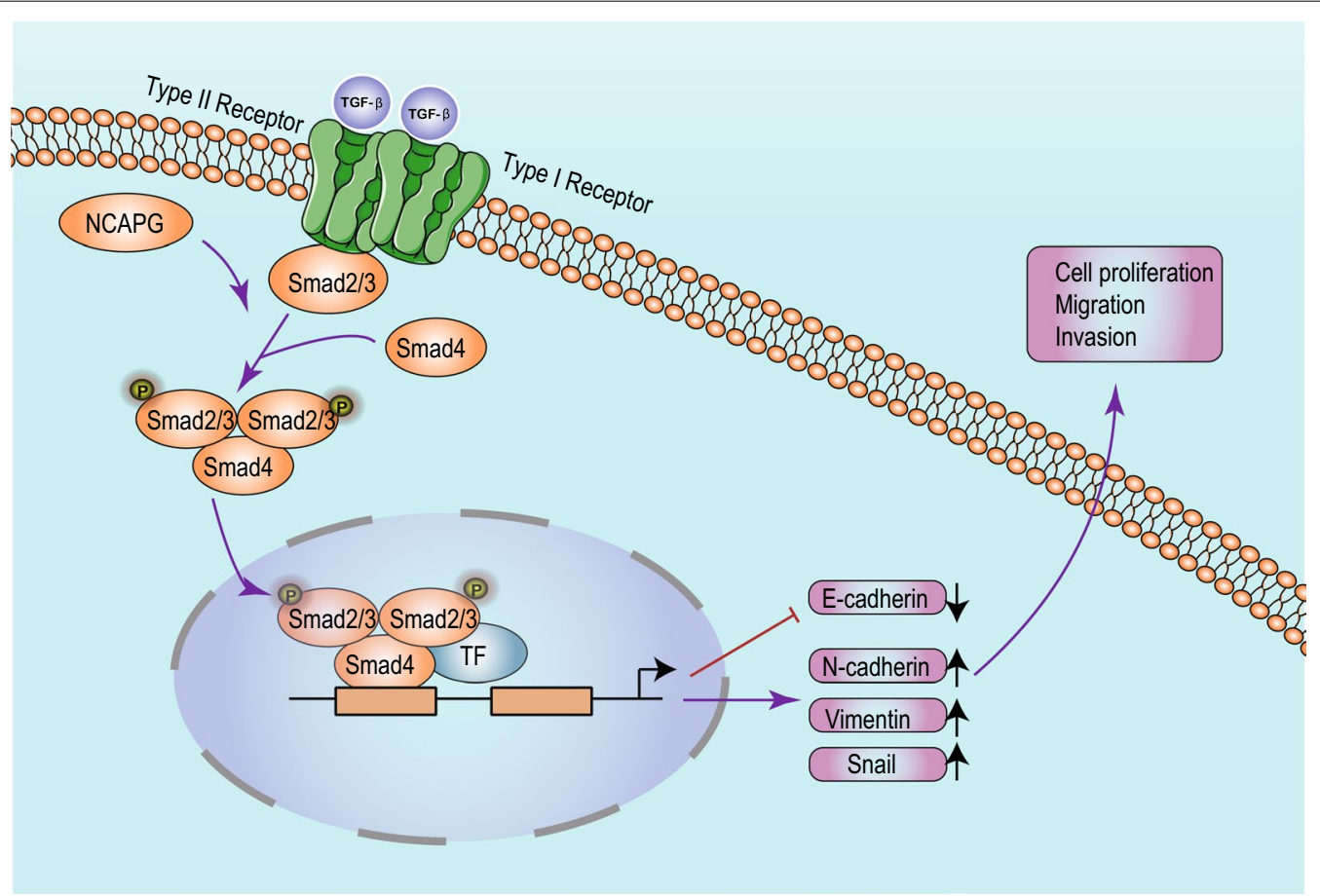

Fig. 10 A proposed model illustrating the function and mechanism of NCAPG in LUAD progression. TF transcription factors

experiments are needed to study the specific mechanism by which NCAPG induces the activation of the TGF- $\beta$ signaling pathway and EMT. Second, previous research on the progress of NCAPG in LUAD-especially its

\section{Abbreviations}

LUAD: Lung adenocarcinoma; NCAPG: Non-SMC condensin I complex, subunit G; TCGA: The Cancer Genome Atlas; DEG: Differentially expressed gene; PPI: Protein-protein interaction; GO: Gene ontology; BP: Biological process; CC: Cell component; MF: Molecular function; KEGG: Kyoto Encyclopedia of Genes; GSEA: Gene set enrichment analysis; CCK8: Cell Counting Kit 8; EMT: Epithelialmesenchymal transition; HCC: Hepatocellular carcinoma; PBS: Phosphate 
buffered saline; shRNA: Short hairpin RNA; TGF- $\beta$ : Transforming growth factor $\beta ;$ CTD: Comparative Toxicogenomics Database.

\section{Supplementary Information}

The online version contains supplementary material available at https://doi. org/10.1186/s12935-021-02138-w.

Additional file 1: Table S1. Antibodies used in this study.

Additional file 2: Table S2. Overlapping differentially expressed genes in the three databases.

Additional file 3: Figure S1. PPI network of overlapping genes.

Additional file 4: Figure S2. Kaplan-Meier overall survival analysis of the top 14 genes in patients with LUAD.

\section{Acknowledgements}

We would like to thank Editage (www.editage.cn) for English language editing.

\section{Authors' contributions}

We would like to thank Dr. Yi Huang for permitting us to carry out our experiments in Center for Experimental Research in Clinical Medicine.

\section{Funding}

This study was supported by the Natural Science Foundation of China (Grant Number: 82002457), Startup Fund for scientific research, Fujian Medical University (Grant Number: 2019QH2045, 2018QH1130), Health research talents training program of Fujian province (Grant Number: 2019-ZQNB-1), and highlevel hospital grants from Fujian Provincial Hospital, Fujian province, China (Grant Number: 2018GSP008).

\section{Availability of data and materials}

The data that support the findings of this study are openly available in The Cancer Genome Atlas (TCGA) data portal (https://tcga-data.nci.nih.gov/tcga/) and Gene Expression Omnibus (GEO) database (https://www.ncbi.nlm.nih. $\mathrm{gov} / \mathrm{geo} /$ ). The rest of the data are available from the corresponding author on reasonable request.

\section{Declarations}

\section{Ethics approval and consent to participate}

The Ethics Committee of Fujian Provincial Hospital approved the use of all human samples. Animal procedures were approved by the Animal Ethics Committee of Fujian University of Traditional Chinese Medicine.

\section{Consent for publication}

Not applicable.

\section{Competing interests}

The authors report no conflicts of interest regarding this work.

\section{Author details}

'Shengli Clinical Medical College of Fujian Medical University, Fuzhou 350001, China. ${ }^{2}$ Department of Pulmonary and Critical Care Medicine, Fujian Provincial Hospital, Fuzhou 350001, China. ${ }^{3}$ Fujian Provincial Researching Laboratory of Respiratory Diseases, Fuzhou 350001, China.

\section{Received: 30 May 2021 Accepted: 3 August 2021}

Published online: 21 August 2021

\section{References}

1. Global Burden of Disease Cancer Collaboration, Fitzmaurice C, Dicker D, Pain A, Hamavid H, Moradi-Lakeh M, et al. The Global burden of cancer 2013. JAMA Oncol. 2015;1:505-27.

2. Travis WD. Lung cancer pathology: current concepts. Clin Chest Med. 2020;41:67-85
3. Travis WD, Brambilla E, Nicholson AG, Yatabe Y, Austin JHM, Beasley MB, et al. The 2015 World Health Organization classification of lung tumors: impact of genetic, clinical and radiologic advances since the 2004 classification. J Thorac Oncol. 2015;10:1243-60.

4. Testa U, Castelli G, Pelosi E. Lung cancers: molecular characterization, clonal heterogeneity and evolution, and cancer stem cells. Cancers. 2018;10:248.

5. Schiller JH, Harrington D, Belani CP, Langer C, Sandler A, Krook J, et al. Comparison of four chemotherapy regimens for advanced non-small-cell lung cancer. N Engl J Med. 2002;346:92-8.

6. Murphy LA, Sarge KD. Phosphorylation of CAP-G is required for its chromosomal DNA localization during mitosis. Biochem Biophys Res Commun. 2008:377:1007-11.

7. Gong C, Ai J, Fan Y, Gao J, Liu W, Feng Q, et al. NCAPG promotes the proliferation of hepatocellular carcinoma through PI3K/AKT signaling. Onco Targets Ther. 2019;12:8537-52.

8. Liu W, Liang B, Liu H, Huang Y, Yin X, Zhou F, et al. Overexpression of nonSMC condensin I complex subunit G serves as a promising prognostic marker and therapeutic target for hepatocellular carcinoma. Int J Mol Med. 2017;40:731-8.

9. Wang Y, Gao B, Tan PY, Handoko YA, Sekar K, Deivasigamani A, et al. Genome-wide CRISPR knockout screens identify NCAPG as an essential oncogene for hepatocellular carcinoma tumor growth. FASEB J. 2019;33:8759-70.

10. Ai J, Gong C, Wu J, Gao J, Liu W, Liao W, et al. MicroRNA181c suppresses growth and metastasis of hepatocellular carcinoma by modulating NCAPG. Cancer Manag Res. 2019;11:3455-67.

11. Song B, Du J, Song DF, Ren JC, Feng Y. Dysregulation of NCAPG, KNL1, miR-148a-3p, miR-193b-3p, and miR-1179 may contribute to the progression of gastric cancer. Biol Res. 2018;51:44.

12. Zhang $X$, Wang $H$, Han $Y$, Zhu M, Song Z, Zhan D, et al. NCAPG induces cell proliferation in cardia adenocarcinoma via PI3K/AKT signaling pathway. Onco Targets Ther. 2020;13:11315-26.

13. Jiang $L$, Ren L, Chen H, Pan J, Zhang Z, Kuang X, et al. NCAPG confers trastuzumab resistance via activating SRC/STAT3 signaling pathway in HER2-positive breast cancer. Cell Death Dis. 2020;11:547.

14. Chen J, Qian X, He Y, Han X, Pan Y. Novel key genes in triple-negative breast cancer identified by weighted gene co-expression network analysis. J Cell Biochem. 2019;120:16900-12.

15. He R, Wang L, Li J, Ma L, Wang F, Wang Y. Integrated analysis of a competing endogenous RNA network reveals a prognostic signature in kidney renal papillary cell carcinoma. Front Cell Dev Biol. 2020;8:612924.

16. Yu G, Wang LG, Han Y, He QY. clusterProfiler: an R package for comparing biological themes among gene clusters. OMICS. 2012;16:284-7.

17. Shannon P, Markiel A, Ozier O, Baliga NS, Wang JT, Ramage D, et al. Cytoscape: a software environment for integrated models of biomolecular interaction networks. Genome Res. 2003;13:2498-504.

18. Chin $\mathrm{CH}$, Chen $\mathrm{SH}, \mathrm{Wu} \mathrm{HH}, \mathrm{Ho}$ CW, Ko MT, Lin CY. cytoHubba: identifying hub objects and sub-networks from complex interactome. BMC Syst Biol. 2014;8(Suppl 4):S11.

19. Ghandi M, Huang FW, Jané-Valbuena J, Kryukov GV, Lo CC, McDonald ER 3rd, et al. Next-generation characterization of the cancer cell line encyclopedia. Nature. 2019;569:503-8.

20. Subramanian A, Tamayo P, Mootha VK, Mukherjee S, Ebert BL, Gillette MA, et al. Gene set enrichment analysis: a knowledge-based approach for interpreting genome-wide expression profiles. Proc Natl Acad Sci USA. 2005;102:15545-50.

21. Davis AP, Grondin CJ, Johnson RJ, Sciaky D, Wiegers J, Wiegers TC, et al. Comparative toxicogenomics database (CTD): update 2021. Nucleic Acids Res. 2021:49:D1138-43.

22. Tang Z, Kang B, Li C, Chen T, Zhang Z. GEPIA2: an enhanced web server for large-scale expression profiling and interactive analysis. Nucleic Acids Res. 2019;47:W556-60.

23. Stephens PJ, Tarpey PS, Davies H, Van Loo P, Greenman C, Wedge DC, et al. The landscape of cancer genes and mutational processes in breast cancer. Nature. 2012;486:400-4.

24. Liu Z, Zhang Y, Dang Q, Wu K, Jiao D, Li Z, et al. Genomic alteration characterization in colorectal cancer identifies a prognostic and metastasis biomarker: FAM83A|IDO1. Front Oncol. 2021;11:632430.

25. Thiery JP. Epithelial-mesenchymal transitions in tumour progression. Nat Rev Cancer. 2002:2:442-54. 
26. Okada T, Sinha S, Esposito I, Schiavon G, López-Lago MA, Su W, et al. The Rho GTPase Rnd1 suppresses mammary tumorigenesis and EMT by restraining Ras-MAPK signalling. Nat Cell Biol. 2015;17:81-94.

27. Du B, Shim JS. Targeting epithelial-mesenchymal transition (EMT) to overcome drug resistance in cancer. Molecules. 2016;21:965.

28. Lu C, Yang Z, Yu D, Lin J, Cai W. RUNX1 regulates TGF- $\beta$ induced migration and EMT in colorectal cancer. Pathol Res Pract. 2020:216:153142.

29. Lee $\mathrm{SH}, \mathrm{Kim} \mathrm{O}, \mathrm{Kim} \mathrm{HJ}$, Hwangbo C, Lee JH. Epigenetic regulation of TGF- $\beta$-induced EMT by JMJD3/KDM6B histone H3K27 demethylase. Oncogenesis. 2021;10:17.

30. Javelaud D, Alexaki VI, Mauviel A. Transforming growth factor-beta in cutaneous melanoma. Pigment Cell Melanoma Res. 2008;21:123-32.

31. Meulmeester E, Ten Dijke P. The dynamic roles of TGF- $\beta$ in cancer. J Pathol. 2011;223:205-18

32. Ko H, So Y, Jeon H, Jeong MH, Choi HK, Ryu SH, et al. TGF- $\beta 1$-induced epithelial-mesenchymal transition and acetylation of Smad 2 and Smad3 are negatively regulated by EGCG in human A549 lung cancer cells. Cancer Lett. 2013:335:205-13.
33. Zi Z, Chapnick DA, Liu X. Dynamics of TGF- $\beta / S m a d$ signaling. FEBS Lett. 2012;586:1921-8

34. Zhang M, Tian J, Wang R, Song M, Zhao R, Chen H, et al. Dasatinib inhibits lung cancer cell growth and patient derived tumor growth in mice by targeting LIMK1. Front Cell Dev Biol. 2020;8:556532.

35. Yang H, Sun B, Xu K, He Y, Zhang T, Hall SRR, et al. Pharmaco-transcriptomic correlation analysis reveals novel responsive signatures to HDAC inhibitors and identifies Dasatinib as a synergistic interactor in small-cell lung cancer. EBioMedicine. 2021;69:103457.

36. Conroy LR, Lorkiewicz P, He L, Yin X, Zhang X, Rai SN, et al. Palbociclib treatment alters nucleotide biosynthesis and glutamine dependency in A549 cells. Cancer Cell Int. 2020;20:280.

\section{Publisher's Note}

Springer Nature remains neutral with regard to jurisdictional claims in published maps and institutional affiliations. 